\title{
PET-Saúde Interprofissionalidade, intervenções na atenção primária: um relato de experiência
}

\author{
TEP-Health Interprofessionality, interventions in primary care: an experience report \\ Interprofesionalidad PET-Salud, intervenciones en atención primaria: un informe de \\ experiencia
}

Wellington Manoel da Silva ${ }^{1,2 *}$, Maria Eduarda da Silva ${ }^{2}$, Thuani Lamenha Costa ${ }^{2}$, Millena Maria Nascimento Silva ${ }^{2}$, Geraldo José Santos Oliveira ${ }^{3}$, Maria Angélica Álvares de Freitas ${ }^{3}$, Nayara Ranielli da Costa $^{2}$, Ana Wladia Silva de Lima² ${ }^{2}$ Viviane de Araújo Gouveia², Silvana Gonçalves Brito de Arruda².

\section{RESUMO}

Objetivo: Relatar a experiência da intervenção interprofissional em saúde, destinada à uma família de alto risco social. Relato da Experiência: Trata-se de um estudo descritivo do tipo relato de experiência, realizado com a participação dos estudantes do projeto "Programa de Educação Tutorial em Saúde Interprofissionalidade" de uma Universidade pública de um município de Pernambuco, Brasil. A amostra do estudo foi composta por uma família, escolhida de forma intencional e não-probabilística. Os dados foram obtidos a partir de visitas domiciliares realizadas à família. Foram utilizados instrumentos como, Escala de estratificação de Risco de Coelho e Savassi, Genograma, Ecomapa e Projeto Terapêutico Singular (PTS). A família apresentou um alto grau de vulnerabilidade social, desta forma foi executado um PTS amplo e holístico, na tentativa de alcançar uma melhora tanto no aspecto de saúde da família quanto no âmbito social. Considerações finais: Os resultados evidenciam que o espaço do Projeto permite aos alunos o aprendizado prático, baseado na diversidade de atividades e nas reflexões sobre a importância do trabalho colaborativo da equipe.

Palavras-chave: Comunicação interdisciplinar, Estratégia de saúde da família, Promoção da saúde.

\begin{abstract}
Objective: To report the experience of interprofessional health intervention, aimed at a family at high social risk. Experience report: Descriptive study of the experience report type, carried out with the participation of students from the project "Tutorial Education in Health Program - Interprofessionality" from a public university in a municipality in Pernambuco, Brazil. The study sample was composed of a family, chosen intentionally and non-probabilistically. Data were obtained from home visits to the family. Instruments such as Coelho and Savassi Risk Stratification Scale, Genogram, Ecomap and Singular Therapeutic Project (STP) were used. The family had a high degree of social vulnerability, so a broad and holistic PTS was performed, in an attempt to achieve an improvement both in the family's health aspect and in the social sphere. Final considerations: The results show that the Project space allows students to learn hands-on, based on the diversity of activities and reflections on the importance of the team's collaborative work.
\end{abstract}

Key words: Interdisciplinary communication, Family health strategy, Health promotion.

\footnotetext{
1 Instituto de Medicina Integral Professor Fernando Figueira (IMIP), Recife - PE.

*E-mail: wellington-manoel@outlook.com

2 Universidade Federal de Pernambuco (UFPE), Vitória de Santo Antão - PE.

${ }^{3}$ Secretaria Municipal de Saúde de Vitória de Santo Antão, Vitória de Santo Antão - PE.
} 


\section{RESUMEN}

Objetivo: Reportar la experiencia de Informar sobre la experiencia de la intervención sanitaria interprofesional, dirigida a una familia con alto riesgo social. Informe de experiencia: Estudio descriptivo del tipo de informe de experiencia, realizado con la participación de estudiantes del proyecto "Tutorial Education in Health Program - Interprofessionality" de una universidad pública en un municipio de Pernambuco, Brasil. La muestra del estudio estaba compuesta por una familia, elegida intencionalmente y no probabilísticamente. Los datos se obtuvieron de visitas domiciliarias a la familia. Se utilizaron instrumentos como la Escala de estratificación de riesgo de Celho y Savassi, Genograma, Ecomap y Proyecto terapéutico singular (PTS). La familia tenía un alto grado de vulnerabilidad social, por lo que se realizó un PTS amplio y holístico, en un intento por lograr una mejora tanto en el aspecto de salud de la familia como en la esfera social. Consideraciones finales: Los resultados muestran que el espacio del Proyecto permite a los estudiantes aprender de manera práctica, basándose en la diversidad de actividades y reflexiones sobre la importancia del trabajo colaborativo del equipo.

Palabras clave: Comunicación interdisciplinaria, Estrategia de salud familiar, Promoción de la salud.

\section{INTRODUÇÃO}

O cenário atual encontra-se marcado por intensas transformações nos campos político, científico, cultural, tecnológico e econômico com crescente complexidade e fragmentação da assistência à saúde. Portanto, a prática Interprofissional ganhou projeção e reconhecimento na promoção à saúde de maneira mais efetiva, segura e integral (ALMEIDA RGS, et al., 2019; ESCALDA P e PARREIRA CMSF, 2018).

O trabalho Interprofissional pode ser definido como uma troca de saberes, entre profissionais de distintos campos disciplinares, que ocorre de forma coesa, envolvendo reflexão e trabalho com potencial resolutivo das necessidades da comunidade (D'AMOUR D e OANDASAN I, 2008).

Embora, os profissionais de saúde possuam processos de trabalho distintos, o trabalho em equipe, caracteriza-se como uma forma de trabalhar coletivamente, e é desenvolvida no cotidiano por meio da comunicação efetiva em busca da integração de conhecimento tendo em vista promover as melhores ações durante o cuidado ao indivíduo (PEDUZZI M, 2010). A comunicação efetiva e o trabalho em equipe são atores de uma assistência em saúde prestada com qualidade e segurança (OLINO L, et al., 2019).

Embora seja uma discussão atual em nosso país, essa temática foi proposta desde 1960 na Europa, especificamente na França e Itália, e é considerada como uma possibilidade de produção de conhecimento na busca por respostas às indagações deixadas pelo modelo hegemônico experimental, centrado em abordagens no plano individual, disfunções orgânicas e práticas curativas (LIMA ACS E AZEVEDO CB, 2013).

Ao reconhecer as limitações em alcançar a integralidade do cuidado, por meio de práticas isoladas e fragmentadas, o conceito de Educação Interprofissional (EIP), surge como estratégia de reorganização do processo de trabalho em saúde, todavia, este, enfrenta ainda inúmeros entraves concernentes a sua implantação nos perfis curriculares dos cursos de graduação em saúde no País (RIOS DRS, et al., 2020).

Este novo modelo de ensino e execução da assistência em saúde à comunidade proposto pela EIP, faz parte de uma busca pelo fortalecimento das políticas públicas de saúde voltadas a reorientar o processo de formação profissional (BRASIL, 2018).

Neste contexto, o projeto de extensão Programa de Ensino Tutorial (PET) Saúde - Interprofissionalidade, cujas características marcantes são, trabalho em equipe fortalecido pela comunicação efetiva e comprometimento com a solução de problemas e a negociação na tomada de decisões possui a intenção de romper com a formação tradicional, possibilitando ao estudante uma formação centrada no usuário/paciente com o objetivo de melhoria da qualidade da assistência prestada (BARR H, 1998 e BATISTA NA, 2012). Para Batista NA (2012) estas políticas indutoras de mudanças no perfil curricular, como o PET Saúde Interprofissionalidade, tem se mostrado como relevantes espaços para o desenvolvimento da EIP no país. 
Desta forma, o presente estudo tem como objetivo relatar a experiência, de um grupo de estudantes de graduação das áreas de Enfermagem, Nutrição, Educação Física e Saúde Coletiva de uma Universidade pública, durante a realização das ações de um plano de ação em saúde, de caráter colaborativo, destinado a uma família de alto risco socioeconômico da área de abrangência de uma Unidade Básica de Saúde (UBS), em um município do Estado de Pernambuco, Brasil.

\section{RELATO DE EXPERIÊNCIA}

O estudo foi realizado em um Unidade Básica de Saúde de um município Pernambucano na região nordeste do Brasil. A pesquisa se deu da observação das atividades realizadas durante a execução de um projeto de extensão universitária intitulado PET-Saúde: Interprofissionalidade, promovido por uma Universidade pública, no período de junho a dezembro de 2019. Participaram da experiência discentes e docentes da instituição, dos cursos de Educação Física, Enfermagem, Nutrição, Ciência Biológicas e Saúde Coletiva além de profissionais da saúde do município, como Enfermeira, Psicóloga, Educador Físico e Residente de Enfermagem, estes, na qualidade de preceptores.

Com a finalidade de implementar a Educação Interprofissional, houve a seleção da família a ser assistida, de forma intencional e não probabilística. A família recebeu a equipe durante quatro visitas domiciliares. As etapas de acolhimento, entrevista e observação sistematizada, durante as visitas, foram importantes no subsídio da coleta de dados para elaboração dos instrumentos de Genograma, Ecomapa e Escala de Coelho FLG e Savassi LCM (2012), além da construção do plano de trabalho da equipe, incluindo o Projeto Terapêutico Singular (PTS).

Após as visitas domiciliares a equipe se reunia em uma UBS para fazer o planejamento das próximas visitas, discutir o caso, e implementar os instrumentos. Na primeira visita domiciliar houve obtenção de dados sociodemográficos através da entrevista com os familiares presentes, em seguida durante a reunião de equipe houve a aplicação da Escala de Estratificação de Risco Familiar Coelho FLG e Savassi LCM (2012).

A segunda visita domiciliar teve o objetivo de adquirir informações com vista a construção do planejamento da assistência interdisciplinar por meio da construção do Genograma e Ecomapa. A terceira visita teve como objetivo adquirir informações suficientes para a construção do Projeto Terapêutico Singular (PTS). Após a elaboração das metas do PTS, a equipe se comprometeu em realizar as articulações necessárias e reavaliálas semanalmente para alcance de resolutividade no prazo de seis meses, pois, esse era o prazo de permanência da equipe no projeto, desta forma, a última visita teve por objetivo avaliação das ações do PTS, objetivos alcançados, limitações e orientações finais à família.

A família era composta por 5 integrantes. Com o objetivo de garantir o princípio da privacidade, os nomes dos usuários foram substituídos por numerais ordinais, assim como, as características antropométricas e os dados referentes a estratificação de risco familiar e referentes ao planejamento e execução da assistência. $A$ moradia da Família A, consiste em uma casa cedida de alvenaria, em condições mínimas de acabamento, com cinco cômodos, cobertos com telha, apresentando baixas condições de higiene, piso sem revestimento cerâmico, com energia elétrica e água encanada, todavia cortada, em uma rua com calçamento e com baixas condições de saneamento básico.

O membro 1 (M1) encontrava-se desempregada e teve duas perdas recentes por falecimento (mãe e avó). O membro 2 (M2) possui curso técnico em informática, vendedor autônomo e faz uso de drogas lícitas. $\mathrm{O}$ membro 3 (M3) frequenta o ensino fundamental. O membro 4 (M4), não frequenta creche. Por fim o membro 5 (M5) Viúva recentemente, ex-companheira da mãe do M1, analfabeta, desempregada. Histórico de saúde desconhecido e faz uso de drogas lícitas. Desta forma, obteve-se da aplicação da escala de estratificação de risco para a família o valor igual a 14 enquadrando a família como Risco Máximo (Tabela 1). 
Tabela 1 - Estratificação de risco familiar da família A; por meio da Escala de Coelho e Savassi, 2012.

\begin{tabular}{ccc}
\hline Dados da ficha A & Escore & Família A \\
\hline Acamado & 3 & 0 \\
Deficiência física & 3 & 0 \\
Deficiência mental & 3 & 0 \\
Baixas condições de saneamento & 3 & 3 \\
Desnutrição (grave) & 3 & 0 \\
Drogadição & 2 & 4 \\
Desemprego & 2 & 6 \\
Analfabetismo & 1 & 1 \\
Menor de seis meses & 1 & 0 \\
Maior de 70 anos & 1 & 0 \\
Hipertensão Arterial Sistêmica & 1 & 0 \\
Diabetes mellitus & 1 & 0 \\
Relação $\quad$ Se maior que 1 & 3 & 0 \\
Morador/Cômodo igual a 1 & 2 & 2 \\
\hline Se menor que 1 & 0 & 0 \\
\hline Total & & 14
\end{tabular}

Fonte: Silva WM, et al., 2020.

O vínculo do $\mathrm{M} 1 \mathrm{com}$ a unidade foi considerado muito forte, também refere ter um bom relacionamento com os vizinhos, superficial com a igreja e não pratica nenhuma atividade física. O membro M2 mostrou-se resistente ao serviço de saúde e foi pouco acessível, por vezes recusou-se a estar presente durante as visitas e desta forma, apenas os dados para construção do Genograma e do Ecomapa puderam ser obtidos, da mesma forma que o membro M5 também foi pouco acessível, sendo assim, ambos não participaram diretamente da construção do PTS familiar. M3 e M4 possuem como laços principais seus pais, contudo M3 possui vínculo muito forte com a escola. Assim, foi possível identificar os padrões de organização desta família e a natureza das relações estabelecidas com o meio, evidenciando os desequilíbrios entre as necessidades e os recursos da família (AGOSTINHO M, 2007). Todos os vínculos familiares estão representados conforme mostram Genograma e o Ecomapa (Figura 1).

Figura 1 - Genograma e Ecomapa da Família A.
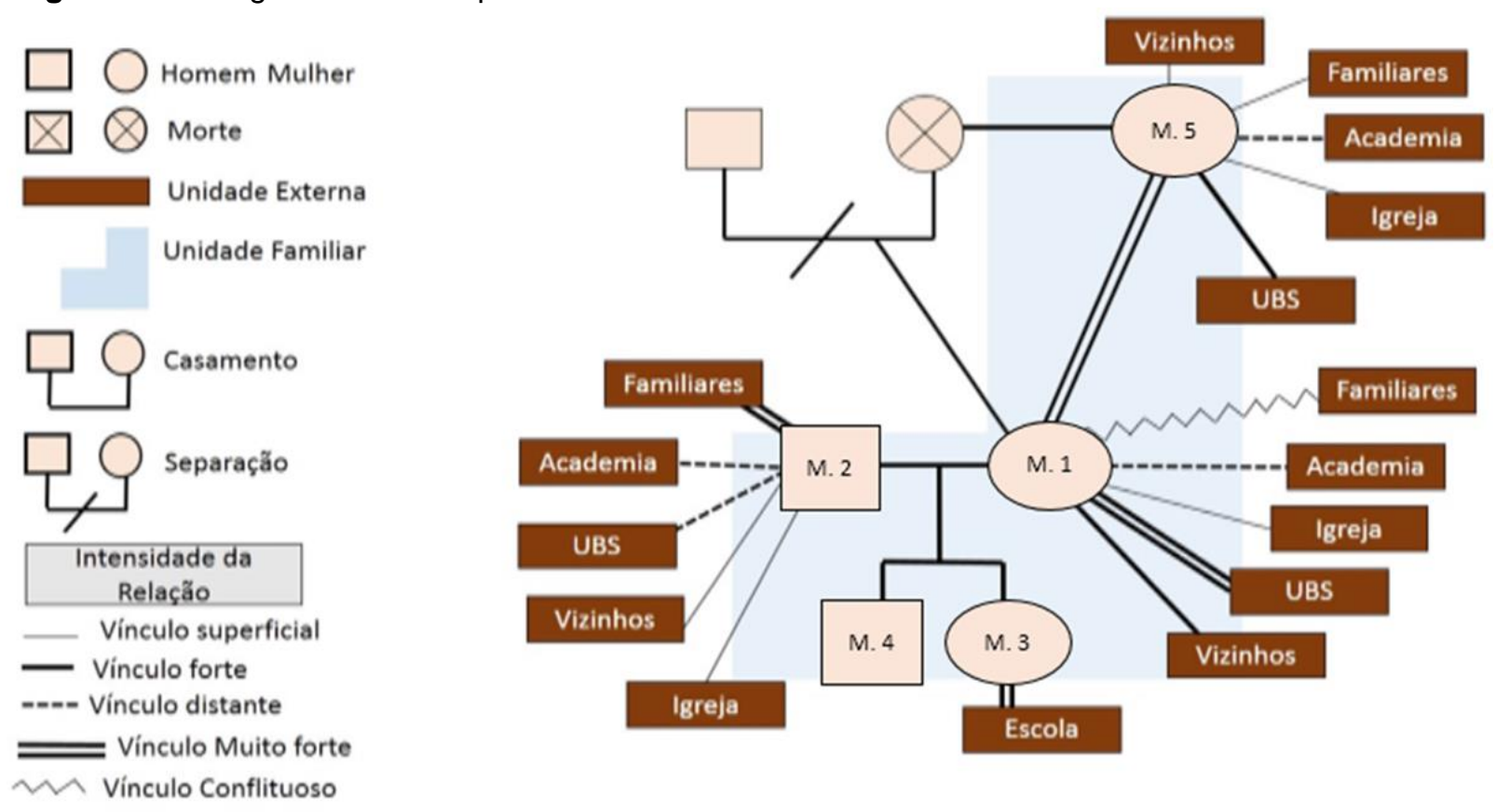

Fonte: Silva WM, et al., 2020. 
O PTS foi elaborado abrangendo os seguintes pontos chave: Referenciar para orientação Técnica psicológica os membros M1, M2 e M5 além de inserir M1 e M5 no Grupo de Mulheres; Empregar Práticas Integrativas e Complementares em Saúde como Ventosaterapia e Auriculoterapia; Atualizar caderneta de vacina da família; Agendar consulta médica para família na UBS; Atualizar dados antropométricos; Inserir o membro no Grupo de Tabagismo; Convidar família a conhecer Projetos de Extensão do Centro Acadêmico, como UFPE; Inserir o membro M2 no grupo de combate ao Tabagismo; Referenciar ao Núcleo Ampliado de Saúde da Família (NASF) para avaliação física e orientações técnicas referente à Educação Física e Psicológica; Realizar orientação nutricional básica de enfermagem; Conscientizar a família da importância do controle de qualidade da água e alimentos; Articular auxílio junto ao Centro de Referência de Assistência Social (CRAS); Investigar possibilidades de formação em cursos profissionalizantes (Assistência social/Entidade Filantrópica); Articular de forma conjunta com a Secretaria Municipal de Educação a inserção do membro M1 na Educação de Jovens e Adultos (EJA).

Devido ao curto prazo de tempo que o projeto possibilitou (seis meses), algumas demandas foram priorizadas, sendo assim foi possível executar: Retirada de possíveis focos de criadouro de larvas de mosquitos; Auxilio no controle de qualidade da alimentação; Incentivo à prática de atividade física; Encorajamento a paciente a participar ativamente de seu cuidado, esclarecendo-a da necessidade de realizar o exame preventivo periodicamente; Houve ainda uma articulação intersetorial junto a Secretaria de Educação, buscando alternativas para uma possível inclusão do membro M1 na EJA; Houve também uma reunião com o CRAS, com o objetivo de examinar se a família se enquadra em maiores benefícios dentro do sistema; Por meio da articulação com o NASF, o membro M1 passou a ser assistido pelo serviço de psicologia.

Visto que muitas dessas ações demandam tempo, a exemplo, a inclusão do membro M1 no EJA, não foi possível acompanhar este processo de integração, embora tenha sido realizado o contato inicial com a Secretaria de Educação. Durante a reunião com a Equipe do CRAS foi identificado também um problema referente à moradia em que a família habita, pois a mesma foi cedida pela avó de M1, enquanto vivia no local, após o falecimento desta, outros parentes estão buscando reaver a casa, desta forma, o membro familiar não informa um endereço fixo ao CRAS impossibilitando o enquadramento da família em qualquer outro tipo de benefício da Assistência Social Municipal.

\section{DISCUSSÃO}

O planejamento da atuação da equipe multiprofissional da Estratégia de Saúde da Família, tendo em vista o princípio da equidade, deve considerar mais que um olhar curativo, visto que, a escolha dos lares a serem assistidos pelas visitas domiciliares, frequentemente, é baseada em função da gravidade da doença do paciente (SAVASSI LCM, 2012). Desta forma, postergando a assistência em saúde às famílias que possuam outro tipo de necessidade relacionada aos condicionantes e determinantes do processo saúde doença, reforçando a necessidade da utilização da Escala de Coelho como ferramenta para apoiar o planejamento das ações de saúde (MENEZES AHR, 2012). A escala, segundo Savassi LCM e Coelho FLG (2012), trata-se de um instrumento utilizado com a finalidade de estratificar o risco familiar, determinando o risco social e de saúde, o que representa a capacidade de adoecimento de cada núcleo familiar. Utiliza-se de sentinelas para a pontuação do risco familiar, com possibilidade de classificação em risco menor (escore total de 5 e 6 ), risco médio (escore total de 7 e 8) ou risco máximo (escore total acima de 9).

Durante o acompanhamento desta família, foi identificado o risco de vulnerabilidade social descrito por Abramovay M (2002), que considera a vulnerabilidade social como uma situação em que a falta de recursos, em um grupo social, impossibilita o mesmo de lidar com as possibilidades ofertadas pela sociedade. Outro fato importante, é que a condição de pobreza vivenciada pela família demonstrou ser um desencadeador de possíveis adoecimentos em alguns membros, tanto gástricos quanto psíquicos. Este fato corrobora com a literatura traz tem mostrado que ambientes mais vulneráveis socioeconomicamente possuem a tendência a ter uma população com saúde precária (SACHS J, 2002; WEIL DN, 2005; SALA-I-MARTIN X, 2005).

Desta forma, foi executado um PTS amplo e holístico, na tentativa de alcançar uma melhora tanto no aspecto de saúde da família quanto no âmbito social. Através do diagnóstico familiar foi possível verificar que 
M1 recebe um destaque por vínculos conflituosos com a família. Walsh $F(2005)$ afirma que, isto pode ser explicado pelo luto vivenciado, pois tais perdas repentinas podem conduzir a um processo de luto mais complexo. Uma situação de luto complicado, geralmente, provoca enorme impacto no sistema familiar e gera processos de enfrentamento distintos para cada indivíduo.

Em concordância com Campos EP (2004), o efeito do suporte familiar ocorre quando o indivíduo percebe o apoio como satisfatório, produzindo nele sentimentos de valorização, compreensão, reconhecimento, acolhimento, proteção e cuidado. E é esse suporte que pode ajudar o indivíduo a enfrentar as dificuldades do cotidiano, trazendo consequentemente respostas positivas para o seu bem-estar.

Neste contexto, o trabalho realizado por Morano CD, et al. (1993), revelou que baixo suporte familiar em momentos de perdas, consiste em um importante preditor de tentativas de suicídio. Desta forma, torna-se necessário a identificação e formação de redes de apoio que atuem como suporte familiar, fator protetor frente às vulnerabilidades. Neste caso, foi observado que os vizinhos estavam atuando como uma rede de apoio ao M1 e consequentemente a família, o que corrobora com o trabalho feito por Faquinello P e Marcon SS (2011), o qual aponta que essa rede alternativa, composta por amigos e vizinhos, é de extrema importância para a promoção da saúde mental.

Conforme é relatado na literatura, a diversidade das necessidades, assim como, sua complexidade demandaram a compreensão e ação colaborativa de toda equipe de saúde (BRASIL, 2008). Os resultados apresentados possibilitaram a identificação de componentes organizacionais da colaboração interprofissional. Considera-se que a proposta trazida pela educação Interprofissional visa proporcionar uma lógica de colaboração profissional sobre a lógica da fragmentação do cuidado, ao disponibilizar recursos organizacionais (espaços coletivos) que possam apoiar o trabalho em equipe (ARRUDA LS e MOREIRA COF, 2018). Os dados obtidos apontaram para a necessária e indispensável troca de informações entre os diversos profissionais, sem a qual, não seria possível delimitar o projeto terapêutico singular da família.

Concernente à proposta de plano assistencial praticada durante o PET Saúde - Interprofissionalidade, visou propiciar aos sujeitos (Família A) e suas necessidades de saúde, lugar central na construção do plano de cuidado, por meio da escuta ativa. Saúde aqui entendida por meio do conceito ampliado, que aponta para o produto resultante das condições de alimentação, habitação, educação, renda, meio ambiente, trabalho, transporte, emprego, lazer, liberdade, acesso e posse da terra e acesso a serviços de saúde advindo da $8^{2}$ conferência nacional de Saúde (Brasil, 1986), desta forma, a ideia de inserir educação profissional no plano assistencial foi reforçada pelo entendimento de empoderamento trazido por Gita S (1997), onde é preciso possuir um maior controle sobre as condições externas e recursos, tais como, materiais, físicos, intelectuais e financeiros para alterar as condições de vulnerabilidade social.

A tríade ensino-serviço-comunidade apresenta-se como importante estratégia para efetiva integração entre teoria e prática. A realização da articulação da universidade com a Estratégia de Saúde da Família proporciona uma maior reflexão aos profissionais e estudantes, que, confrontados com as necessidades da comunidade, aprendem e produzem conhecimentos. Os resultados do presente Relato de Experiência podem contribuir com o debate sobre o trabalho interprofissional, considerando este tema como um recurso estratégico de reestruturação da formação acadêmica dos futuros profissionais de saúde, transformação do modelo assistencial em saúde no Brasil, em especial, na área de Saúde da Família e Comunidade, fortalecendo a Atenção Básica.

\section{REFERÊNCIAS}

1. AGOSTINHO M. Ecomapa. Dossier Família. $2007 . \quad$ Disponível em: <http://www.apmcg.pt/files/54/documentos/20071001155345624718.pdf>. Acesso em: 30 abr. 2020.

2. ABRAMOVAY M, et.al. Juventude, violência e vulnerabilidade social na América Latina: desafios para políticas públicas. Brasília: UNESCO, 2002; 184p.

3. ALMEIDA LHRB, et al. Ensinando e aprendendo com portadores de esclerose múltipla: Relato de experiência. Revista Brasileira de Enfermagem, 2007; 60(4), 460-463.

4. ALMEIDA RGS, et al. A interface entre o PET-Saúde/ Interprofissionalidade e a Política Nacional de Educação Permanente em Saúde. Saúde Debate, 2019; 43(1), 97-105 
5. ARRUDA LS, MOREIRA COF. Interprofessional collaboration: a case study regarding the professionals of the Care Center for Elderly. Interface. 2018; 22(64):199-210.

6. BARR HB. Competent to collaborate; towards a competency-based model for interprofessional education. Journal interprofessional care, 1998; 12(2), 181-188.

7. BATISTA NA. Educação Interprofissional em Saúde: Concepções e Práticas. Caderno FNEPAS, $2012 ;$ (2), $25-28$.

8. BRASIL. Ministério da Saúde. Secretaria de Atenção à Saúde. Departamento de Atenção Básica. Núcleo de Apoio à Saúde da Família - Volume 1: Ferramentas para a gestão e para o trabalho cotidiano. Cadernos de Atenção Básica, n³9. Brasília, 2014; 116 p.

9. BRASIL. Ministério da Saúde. Secretaria de Atenção à Saúde. Núcleo Técnico da Política Nacional de Humanização. Clínica ampliada, equipe de referência e projeto terapêutico singular / Ministério da Saúde, Secretaria de Atenção à Saúde, Núcleo Técnico da Política Nacional de Humanização - 2. ed. - Brasília: Ministério da Saúde, 2008. Disponível em: <http://bvsms.saude.gov.br/bvs/publicacoes/clinica_ampliada_equipe_referencia_2ed_2008. pdf>. Acesso em: 30 abr. 2020.

10. BRASIL. Ministério da Saúde. Secretaria de Gestão do Trabalho e da Educação na Saúde. Departamento de Gestão da Educação na Saúde. Política Nacional de Educação Permanente em Saúde: o que se tem produzido para o seu fortalecimento? Volume 1; 2018: 41-61.

11. CAMPOS EP. Suporte Social e Família. Em: J. Mello Filho (Org.), Doença e família. São Paulo: Casa do Psicólogo, 2004; 347p

12. D'AMOUR D, OANDASAN I. A model and tipology of collaboration between professional in healthcare organization. BMC Health serv. Res., 2008; (8), 188.

13. ESCALDA P, PARREIRA CMSF. Dimensões do trabalho interprofissional e práticas colaborativas desenvolvidas em uma unidade de básica de saúde, por equipe de Saúde da Família. Interface, 2018; 22(2) 1717-27.

14. FAQUINELLO P E MARCON SS. Amigos e vizinhos: uma rede social ativa para adultos e idosos hipertensos. Revista da Escola de Enfermagem da USP, 2011, 45(6): 1345-1352.

15. FRACOLLI LA, BERTOLOZZI MR. Ministério da Saúde. Secretaria de Atenção à Saúde. Departamento de Atenção Básica. Núcleo de Apoio à Saúde da Família. O perfil epidemiológico na prática do enfermeiro no programa de saúde da família. Manual de enfermagem, 2001; 225p.

16. LIMA ACS; AZEVEDO CB. A interdisciplinaridade no Brasil e o ensino de história: um diálogo possível. Revista Educação e Linguagens, 2013; 2(3): 23p.

17. MENEZES AHR, et al. Classificação do risco familiar segundo Escala de Coelho e Savassi - Um relato de experiência. Londrina, Ciência, Cuidado e Saúde. 2012; 11(1): 190-195.

18. MORANO CD, et al. Risk factors for adolescent suicidal behavior: Loss insufficient familial support and hopelessness. Adolescence, 1993, 28(112): 851-865.

19. OLINO L, et al. Comunicação efetiva para a segurança do paciente: nota de transferência e Modified Early Warning Score. Revista Gaúcha de Enfermagem. 2019, (40): e20180341.

20. PEDUZZI M. Trabalho em equipe de saúde no horizonte normativo da integralidade, do cuidado e da democratização das relações de trabalho. ABRASCO. 2010; 21(2): 629-646.

21. RIOS DRS, et al. Diálogos interprofissionais e interdisciplinares na prática extensionista: o caminho para a inserção do conceito ampliado de saúde na formação acadêmica. Interface, 2020; (23): e180080

22. SACHS J. "Macroeconomics and Health: Investing in Health for Economic Development", report of the Commission on Macroeconomics and Health work at the Institute for Global Health. 2002.

23. SAVASSI LCM, et al. Sistematização de um instrumento de estratificação de risco familiar: escala de risco familiar de Coelho-Savassi. Journal of Management and Primary Health Care, 2012; (2), 179-185.

24. SALA-I-MARTIN X. Health and Economic Growth Findings and policy implications. In LÓPEZ-CASANOVA; G; RIVERA, B; CURRAIS, L (eds.): Health and Economic growth: findings and policy implications. 2005.

25. SEN, G. "Empowerment as an approach to poverty" Working Paper series number 97.07 (background paper to the human development report 1997) mimeo, 1997.

26. WALSH F. Fortalecendo a resiliência familiar. São Paulo: Roca, 2005.

27. WEIL DN. "Economic Growth", United States of America: PEARSON. 2005. 